\title{
Factores psicosociales relacionados con la rehospitalización de pacientes con psicosis orgánica
}

\author{
María Teresa Cortés-Padilla, M en Psic, ${ }^{(1)}$ María Luisa Rascón-Gasca, D ra en Psic. ${ }^{(2)}$
}

\begin{abstract}
Cortés-Padilla MT, Rascón-Gasca ML. Factores psicosociales relacionados con la rehospitalización de pacientes con psicosis orgánica Salud Publica Mex 2001:43:529-536. El texto completo en inglés de este artículo está disponible en: http://www.insp.mx/salud/index.html
\end{abstract}

\begin{abstract}
Resumen
Objetivo. Conocer los factores psicosociales relacionados con la frecuente rehospitalización de pacientes con psicosis orgánica. Material y métodos Estudio de tipo observacional descriptivo, de corte transver sal. La muestra estuvo formada por 33 pacientes del Hospital Psiquiátrico Guillermo Dávila, con U nidad de Medicina Familiar N úmero 10 del Instituto Mexicano del Seguro Social de la Ciudad de México, con psicosis orgánica y quienes durante los años de 1993-1994 presentaron mas de dos rehospitalizaciones. Instrumentos: Entrevista grabada, o muestra de conversación de cinco minutos, para la emoción expresada (EE), cuestionarios mixtos para la Concepción de Enfermedad y la Relación médico-paciente. La validez y confiabilidad de los datos se establecieron con las pruebas de Kappa y Alpha de Cronbach y se efectuó análisis estadístico descriptivo e inferencial. Resultados. El $60.9 \%$ de los familiares presentan alto nivel de EE, es decir, expresan crítica, hostilidad 0 sobreinvolucramiento emocional; el $64.3 \%$ de los sujetos de la muestra conviven más de 35 horas a la semana con familiares que presentan Alta EE. Conclusiones. Altos niveles de EE estuvieron asociados con los frecuentes reingresos a hospitalización. Existe mayor conocimiento de las características de la enfermedad por parte del familiar que del paciente. La relación médico-paciente es satisfactoria pero no forma conciencia de enfermedad ni propicia apego al tratamiento terapeútico. El texto completo en inglés de este artículo está disponible en: http:// www.insp.mx/salud/index.html
\end{abstract}

Palabras clave: trastornos organo mentales psicóticos; actitud; emociones; reingresos; México

\section{Cortés-Padilla MT, Rascón-Gasca ML. Psychosocial factors associated with hospital readmissions of patients with organic psychoses \\ Salud Publica Mex 2001;43:529-536. \\ The English version of this paper \\ is available at: http://www.insp.mx/salud/index.html}

\begin{abstract}
A bstract
Objective.To assess the relationship between psychosocial factors, frequent relapses and hospital readmissions in patients with organic psychoses. Material and Methods. A cross-sectional descriptive study was conducted among 33 patients diagnosed with organic psychosis, seen at Hospital Psiquiatrico G uillermo Davila, of Instituto Mexicano del Seguro Social in Mexico City. Patients had been readmitted more than two occasions during 1993-1994. Data collection instruments consisted of recorded interviews, fiveminute dialogues of expressed emotions (EE), and combined questionnaires to assess perception of illness and the physician-patient relationship. The Kappa statistic and Cronbach's alpha were used to establish the validity and reliability of the measurements; descriptive and inferential methods were used for statistical data analysis. Results. A high percentage $(60.9 \%)$ of patient's relatives showed high level of $E E$, as measured by their expression of criticism, hostility, or emotional overinvolvement; $64.3 \%$ of study subjects lived for more than 35 hours per week with relatives having high EE levels. Conclusions High EE levels were associated with frequent readmissions to the psychiatric ward. G reater perception of illness characteristics was observed among relatives than in patients. Even though a satisfactory physician-patient relationship was found, it was not conducive to improving the perception of illness nor compliance with therapy.The English version of this paper is available at: http://www.insp.mx/salud/index.html
\end{abstract}

Key words: organic mental disorders psychotic; attitude; emotions; relapses; Mexico

(1) Hospital Psiquiátrico con Unidad de Medicina Familiar N 0. 10 Instituto Mexicano del Seguro Social. D epartamento de Investigaciones Psicosociales, Instituto $\mathrm{N}$ acional de Psiquiatría Ramón de la Fuente, México, D.F., México.

(2) Instituto Nacional de Psiquiatría Ramón de la Fuente. Dirección de Estudios Epidemiológicos y Sociales, México.

Fecha de recibido: 31 de mayo de 2000 - Fecha de aprobado: 16 de agosto de 2001

Solicitud de sobretiros: D ra. María Luisa Rascón, Instituto N acional de Psiquiatría Ramón de la Fuente. Calzada México-X ochimilco \#101 colonia San Lorenzo Huipulco, D elegación de Tlalpan, 14370 México, D.F., México.

Correo electrónico: rascong@ imp.edu.mx 
$E$ n los casos de trastornos mentales lo suficientemente severos como para requerir hospitalización existe una prevalencia mayor en los hombres (5.4\%) que en las mujeres $(4.1 \%)$, y por lo que respecta a las psicosis, la diferencia entre ambos sexos es muy pequeña, siendo ligeramente más frecuente en la población masculina. ${ }^{1}$ Los pacientes que formaron la muestra de esta investigación fueron seleccionados por tener el diagnóstico de psicosis orgánica y por haber presentado más de dos ingresos a hospitalización durante los años de 1993 y 1994; en su mayoría fueron hombres, y la psicosis orgánica que padecen fue inducida por traumatismo craneoencefálico, alcoholismo crónico y farmacodependencia, fenómenos más frecuentes en el género masculino; la mayoría de estos enfermos son crónicos ya que su padecimiento no es curable sino controlable, lo que implica que estén utilizando los servicios e instalaciones psiquiátricas periódicamente y en algunos casos los reingresos exceden a los que corresponden a la evolución propia de la enfermedad.

Para la familia el pariente enfermo es un integrante que trastorna la dinámica familiar, que mueve los afectos en forma polarizada: por un lado puede obtener una sobreprotección que lo hace sumamente dependiente y lo incapacita más, y por otro, obtiene el rechazo total de la familia, de tal manera que lo quieren depositar permanentemente en la institución. Cuando existe un miembro en la familia con este trastorno se observa una alteración importante dentro de ella. Las características clínicas de esta enfermedad generan un gran número de situaciones negativas como dificultad en las relaciones familiares, empobrecimiento en la calidad de vida, aislamiento social que interfiere en el funcionamiento laboral, escolar y en el propio hogar, lo que aumenta el nivel de estrés ya existente, convirtiéndose en un factor adicional de angustia, tanto para el paciente como para su familia, llegando a ser considerado como precipitante de recaídas, de exacerbación de síntomas y de rehospitalizaciones. Es evidente que los integrantes de la familia requieren ayuda y apoyo para sobrellevar el manejo de estos pacientes tanto en la casa como en el hospital.

Para la institución los reingresos representan un problema debido a que la atención que recibe el paciente, los esfuerzos realizados para lograr su recuperación y los gastos inicialmente invertidos son inútiles pues el paciente se encuentra nuevamente enfermo y, peor aún, cada vez más deteriorado.

Los frecuentes reingresos al hospital, nos hacen pensar que existen factores que intervienen, no sólo para evitar el control de la enfermedad del paciente, sino para facilitar sus constantes recaídas. Estos fac- tores pueden estar presentes en el ambiente familiar sobre todo en la emoción expresada por el familiar más cercano al paciente, o sea, las actitudes y conductas de éste hacia el enfermo, las cuales según varios estudios están asociadas con un alto riesgo de recaídas. ${ }^{2}$

El concepto de EE proviene de los trabajos iniciados en Inglaterra, cuyas técnicas de medición confiables y estudios relacionados con la validez dieron como resultado la Entrevista Camberwell (CFI) "Entrevista Familiar Armoniosa", técnica con la cual se valoran los niveles de emoción expresada por el familiar, clasificándolos como familiares con Alta o Baja EE. ${ }^{3}$ Con el paso del tiempo estos estudios se extendieron a otros países y la entrevista fue teniendo una serie de modificaciones tendientes a mejorar formas de aplicación y codificación; de esta manera surge la "Muestra de Conversación de Cinco Minutos" (FMSS) la cual originalmente fue desarrollada por Gottschalk y Gleser. ${ }^{4}$ La medida se deriva de los comentarios hechos por un familiar clave del paciente en un periodo de cinco minutos, es decir que con este sistema de codificación se identifican las emociones, los sentimientos y actitudes expresados por una persona acerca de un miembro de su familia mentalmente enfermo.

En un estudio se concluye que los pacientes con mayor gravedad clínica tienen familiares más críticos y hostiles, y que los pacientes con peor ajuste social viven con familias más críticas. Y esto se podría interpretar como que el nivel de crítica y la hostilidad por parte del familiar podrían ser consecuencia de la situación clínica del paciente, y a su vez una causa de ésta. ${ }^{5}$

El desconocimiento y la no aceptación de la enfermedad como parte de la concepción de la misma, tanto del familiar como del paciente, es otro factor importante que origina abandono del tratamiento, pues ni los familiares aceptan la enfermedad de su paciente, ni los pacientes aceptan tener una enfermedad incurable que requiere tomar medicamentos de por vida; ante esta negación, y con las fantasías de curarse, en cuanto se sienten bien dejan de tomarlos, o también cuando creen que los medicamentos les están haciendo "daño" por los efectos secundarios que originan, según informes dados por los pacientes y sus familiares al ingresar al hospital. Durante la práctica clínica la observación de este fenómeno despertó el interés para llevar a cabo esta investigación.

Algunos estudios sobre las atribuciones hacia la enfermedad mental se caracterizan por no compasión, poco afecto, enojo, no ayuda (afecto negativo). Cuando se proporciona información pocos estigmas o juicios de valor se modifican y los que cambian producen afecto positivo, manifestándose como compasión, amor, ayuda y apoyo. ${ }^{6}$ 
Diferentes valores y creencias familiares pueden hacer que algunos individuos sean más tolerantes y menos críticos a las conductas extrañas. En las sociedades latinas la familia es a menudo considerada la más importante unidad social. ${ }^{7}$ Se ha observado que personas con fuertes lazos familiares pueden responder a conductas disruptivas de un ser querido de un modo más benigno a fin de preservar los lazos familiares; esto explica en parte los diferentes niveles de EE observados. ${ }^{8}$ Asimismo, la percepción de cohesión familiar estuvo relacionada inversamente con afecto negativo. Es decir, al incrementar la cohesión familiar se observó decremento en la expresión de sentimientos negativos hacia un paciente con esquizofrenia. ${ }^{9}$

La relación médico-paciente es otro factor importante dado que de ella depende que el paciente tenga una correcta concepción de enfermedad, un apego al tratamiento farmacológico y por ende lo lleve con éxito. ${ }^{10}$ Generalmente en nuestro medio al médico se le considera la persona que alivia los padecimientos, por ello se establece una relación asimétrica en donde el médico tiene los conocimientos y por lo tanto el poder y la autoridad suficientes para influir en quien, debido a sus dolencias, solicita su intervención y por tanto se somete al tratamiento que éste le indique.

Obviamente que el médico no debe tomar esta posición de omnipotencia para dirigirse a sus pacientes, sino establecer con ellos una relación empática en donde el paciente, por el deseo de mejorar y por no deteriorar esa relación, acatará todas las disposiciones emanadas de quien se interesa por su salud.

Con esta ventaja a su favor el médico puede influir de tal manera que pueda lograr un apego al tratamiento farmacológico, una disposición enorme para curarse y sobre todo, en los enfermos crónicos en los cuales la enfermedad sólo será controlada, podrá hacer conciencia de enfermedad que tanta falta hace y lograr que acepten su padecimiento y la toma de medicamentos de por vida. ${ }^{11}$

Llevando con éxito el tratamiento, el médico podrá estar seguro de que su intervención se llevó a cabo en forma eficaz, que logró el control de la enfermedad y que su paciente está en posibilidad de reincorporarse a sus actividades laborales hasta donde sus capacidades se lo permitan, y, sobre todo, mejorar un poco su calidad de vida. Esta es la importancia de una buena relación médico-paciente. ${ }^{12}$

El objetivo de este trabajo fue investigar si la emoción expresada del familiar más cercano al paciente, la concepción de enfermedad que tienen ambos y la relación médico-paciente son factores que se relacionan con los frecuentes reingresos a hospitalización.

\section{Material y métodos}

Este estudio transversal cualitativo y cuantitativo se llevó a cabo en el hospital psiquiátrico con Unidad de Medicina Familiar No. 10 del Instituto Mexicano del Seguro Social.

Los sujetos de la muestra fueron seleccionados de acuerdo con los siguientes criterios de inclusión-exclusión:

De inclusión: a) pacientes de ambos sexos; b) con edad de 18 a 65 años; c) con dos o más reingresos hospitalarios durante los años de 1993 y 1994; d) con psicosis orgánica; e) con expediente vigente en consulta externa; f) que el familiar y el paciente convivan y estén dispuestos a colaborar.

De exclusión: a) pacientes con varios reingresos hospitalarios que no tuvieron el diagnóstico de psicosis orgánica; b) pacientes con retraso mental ; c) pacientes analfabetos; d) pacientes que no desearan colaborar, y e) pacientes cuyo familiar más cercano no quisiera colaborar.

La muestra quedó formada por 33 pacientes ( 23 hombres y 10 mujeres) cuyo número de hospitalizaciones durante el periodo de estudio aparece con los siguientes rangos:
De 2 a 4 hospitalizaciones

De 5 a 7 hospitalizaciones

De 8 a 11 hospitalizaciones

De 12 o más hospitalizaciones
15 pacientes

9 pacientes

5 pacientes

4 pacientes
Para recabar la información se utilizaron cinco instrumentos de los cuales dos se aplicaron al familiar más cercano y tres al paciente.

1. La muestra de conversación de cinco minutos ${ }^{2}$ es una entrevista semiestructurada aplicada al familiar más cercano al paciente; consiste en grabar un monólogo que dura cinco minutos en el que el familiar expresa las emociones, sentimientos y actitudes respecto del paciente. Su validación y confiabilidad se hizo entre jueces: Kappa: 0.7534 y Alpha: $0.8570^{*}$ Rascón y colaboradores.*

2. El cuestionario Conocimiento de la enfermedad del familiar (CONENFA) es mixto y consta de 41

\footnotetext{
* Resultados presentados en la XV Reunión de Investigación del Instituto Nacional de Psiquiatría Ramón de la Fuente, con el tema: Análisis de la técnica del discurso de cinco minutos y su utilidad para evaluar interacción familiar en pacientes con esquizofrenia. Septiembre 20, 2001.
} 
items dispuestos en tres áreas: la primera corresponde a datos demográficos; la segunda investiga la actitud del familiar ante la enfermedad mental del paciente y la tercera investiga el conocimiento de la enfermedad, así como la importancia que le da a los medicamentos. En el análisis cuantitativo este instrumento obtuvo un Alpha de Cronbach de 0.42 , por lo que se consideraron necesarias una revisión y restructuración de los reactivos; sin embargo, los resultados que arrojó fueron muy interesantes y respondieron a los objetivos planteados en este trabajo.

3. El cuestionario Opinión afectiva (OPAF) es mixto y consta de 23 reactivos; fue aplicado al paciente para conocer la opinión afectiva que tiene respecto de su familiar, el tiempo que convive con él, cómo es esa convivencia, con quién tiene más conflicto y cómo se siente en su ambiente familiar. En el análisis obtuvo un Alpha de Cronbach de 0.78

4. El cuestionario Conocimiento de enfermedad del paciente (CONENFPA) es mixto y consta de 18 reactivos y con el cual se investigó el conocimiento que tiene el paciente de su enfermedad y su idea acerca de los medicamentos; el Alpha de Cronbach que obtuvo este instrumento fue de 0.86

5. El cuestionario Relación médico-paciente es mixto y consta de 18 reactivos; con él se investigó la calidad del servicio que presta la institución, la información que el paciente tiene de su enfermedad y su relación con el psiquiatra; el Alpha de Cronbach de este instrumento fue de 0.69 .

La muestra se obtuvo de las estadísticas que obran en el archivo de la clínica, con revisión minuciosa de los expedientes de cada uno de los pacientes que cubrían los criterios de inclusión. Inicialmente los instrumentos se aplicaron en un cubículo del hospital y por circunstancias ajenas al estudio fue necesario aplicarlos en el domicilio de los pacientes. Debido a la información recabada fue necesario introducir dos tipos de análisis de resultados:

Cualitativo: con la información grabada de la FMSS fue necesario utilizar el manual de clasificación, ${ }^{2}$ el cual está dividido en cuatro categorías que son: 1) concepto inicial, 2) relaciones, 3) crítica y 4) sobreinvolucramiento emocional; estas categorías se dividen en subcategorías; el proceso se realizó de la siguiente manera: a) trascripción de las entrevistas, b) análisis del contenido mediante las categorías establecidas y posteriormente c) la codificación, que determina el alto o bajo nivel de emoción expresada por el familiar; el análisis de esta variable se hizo con una muestra de 23 pacientes debido a que fueron anuladas 10 grabaciones por fallas en el equipo.

Cuantitativo: con la información recabada en los cuatro cuestionarios: CONENFA, OPAF, CONENFPA y relación médico-paciente, cuya confiabilidad ya fue mencionada, se hizo análisis estadístico descriptivo e inferencial.

\section{Resultados}

Los datos demográficos muestran que $69.7 \%$ de los sujetos fueron hombres y $30.3 \%$ mujeres, con edad promedio de 34.5 años. El $78.7 \%$ de los pacientes era soltero.

Por lo que respecta a los familiares que se hacen cargo de los pacientes $90.9 \%$ son mujeres y de este porcentaje $51.5 \%$ corresponde a la madre, con una edad promedio de 51 años y escolaridad de primaria.

El $39.1 \%$ de pacientes viven con familiares que presentaron bajo grado de (EE) y para $60.9 \%$ sus familiares tuvieron alto grado de EE (cuadro I).

Al hacer la correlación entre la emoción expresada del familiar y el tiempo que convive el paciente con éste se observó que $64.3 \%$ de los familiares que conviven todo el día con su paciente presentan alto nivel de $\mathrm{EE}$, en contraste con $66.7 \%$ de parientes que nunca han convivido durante todo el día con su paciente y cuya EE es baja (figura 1).

En cuanto al trato que su familia le brinda 55.5\% de los pacientes consideran que es siempre positivo, $31.5 \%$ lo perciben así sólo de vez en cuando y 11\% afirman que nunca reciben buen trato. En cuanto a la percepción que el paciente tiene de su ambiente familiar $48.5 \%$ la reconocen como positiva y $32.1 \%$ consideran que de vez en cuando es negativa. Los sentimientos que ese ambiente le producen al paciente para $42.4 \%$ son positivos, para $39.4 \%$ son positivos de vez en cuando y $10.1 \%$ los considera negativos.

El $90.9 \%$ de los familiares saben que a pesar de estar controlada la enfermedad pueden presentarse recaídas; $72.7 \%$ conocen que la enfermedad es incurable y que sólo podrá ser controlada; $66.7 \%$ saben que su paciente no se curará totalmente tomando los medicamentos. Se observaron diferencias significativas entre $\left(\chi^{2}: 14652\right.$, gl $\left.4, p<0.005\right) ; 54.5 \%$ de los familiares sí conocen las características de la enfermedad de su paciente. Se observaron diferencias significativas al hacer el cruce entre las variables $\left(\chi^{2}: 18\right.$ 861, gl 4, $p<0.001$ ) (cuadro II).

Tanto los familiares como los pacientes en su mayoría desconocen el nombre de la enfermedad y así vemos que $21.2 \%$ de los familiares se refieren a psicosis orgánica pero $39.5 \%$ no saben; por lo que respecta a 


\section{Cuadro I}

EMOCIÓN EXPRESADA POR EL FAMILIAR DEL PACIENTE.

México, D.F., Instituto Mexicano del Seguro Social, 1993-1994

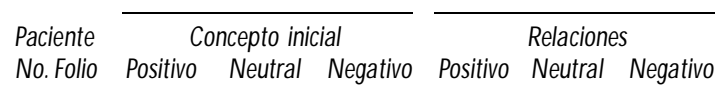

\begin{tabular}{|c|c|c|c|c|c|c|c|c|c|c|c|c|c|}
\hline 1 & & & $1 *$ & & 2 & 2 & 2 & & 2 & & & 1 & Alta \\
\hline 2 & & 1 & & & & 1 & & 3 & 2 & & & & Alta \\
\hline 3 & & 1 & & & 3 & 1 & 1 & 1 & & 1 & & & Alta \\
\hline 7 & & 1 & & & 3 & 1 & & 1 & & 2 & & & Alta \\
\hline 8 & & 1 & & & 4 & 2 & 4 & & & & & & Alta \\
\hline 9 & & 1 & & & 3 & 1 & 1 & & & & & & Alta \\
\hline 10 & & 1 & & & 1 & & & & & 2 & & & Baja \\
\hline 12 & & 1 & & & 1 & & & & & & 1 & 1 & Baja \\
\hline 13 & & 1 & & & 4 & & 1 & & 2 & & & 1 & Alta \\
\hline 14 & 1 & & & 2 & 3 & & & & & & 1 & & Baja \\
\hline 15 & & 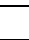 & 1 & & & 2 & 1 & 1 & & & & & Alta \\
\hline 18 & & 1 & & & 1 & & 1 & 1 & 1 & & & & Alta \\
\hline 19 & & 1 & & & & & & & & & & & Baja \\
\hline 20 & & 1 & & & & 2 & 2 & 2 & 2 & & & & Alta \\
\hline 24 & & 1 & & & & & 1 & & 1 & & & & Alta \\
\hline 25 & & 1 & & & 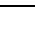 & & & & 1 & & & & Baja \\
\hline 27 & & 1 & & 3 & 2 & & & & & & 1 & & Baja \\
\hline 28 & & 1 & & & 2 & & & & 1 & & & & Baja \\
\hline 29 & & & 1 & & 2 & 3 & 10 & & & & & & Alta \\
\hline 30 & 1 & & & 2 & 3 & 1 & & & & & & & Baja \\
\hline 31 & & 1 & & & & 4 & 3 & & 4 & & & & Alta \\
\hline 32 & & 1 & & 2 & & & & & & & & & Baja \\
\hline 33 & & 1 & & & & & 1 & & 3 & & & & Alta \\
\hline
\end{tabular}

*C ada número indica las veces que se presentó la situación que en el encabezado se señala

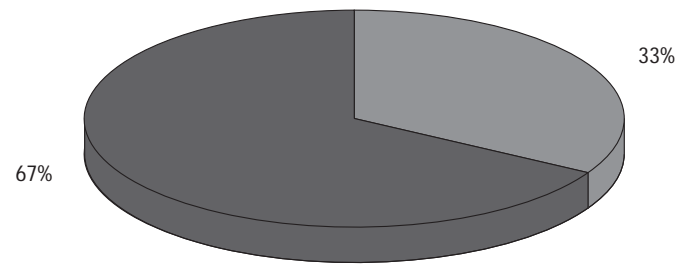

Baja E.E.

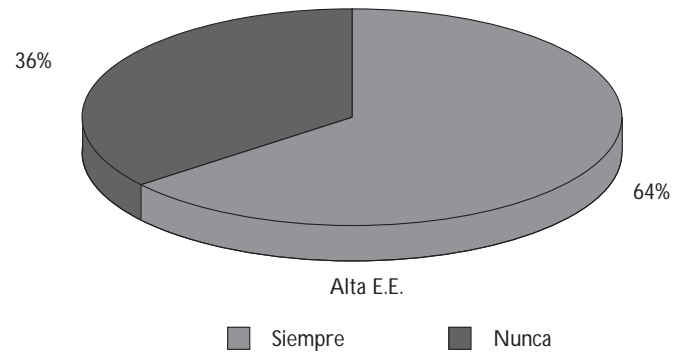

${ }^{*}$ Porcentajes tomados del total de familiares por tipo de emoción expresada

Figura I. EMOción EXPRESADA DEL FAMILIAR QUE CONVIVE todo el día Con el PaCIENTE. ${ }^{*}$ México, D.F., Instituto Mexicano del Seguro Social, 1993-1994 los pacientes, $18.2 \%$ sí conocen el nombre de su padecimiento y $51.5 \%$ no lo conocen, el resto de familiares y de pacientes indicaron el nombre de otros padecimientos.

El $54.5 \%$ de los pacientes considera que su enfermedad es curable; un porcentaje similar cree que volverán a estar como antes de enfermarse. Al hacer el cruce de estas dos variables se obtuvo $\left(\chi^{2}=7200, \mathrm{gl} 1\right.$, $p<0.007$ ) (cuadro III).

El 51.5\% de los pacientes creen que tomando los medicamentos se curarán totalmente, $21.2 \%$ no lo saben, $36.4 \%$ consideran que los medicamentos no los deberán tomar de por vida (cuadro III).

Los resultados obtenidos con el cuestionario relación médico-paciente nos dan a conocer que $84.8 \%$ de los pacientes reciben su consulta cada mes y el tiempo que dura dicha consulta es entre cinco minutos y tres cuartos de hora, tiempo que $66.7 \%$ de los pacientes consideran suficiente; $72.7 \%$ de los sujetos de la muestra ha sido atendido hasta por tres psiquiatras y $69.7 \%$ opina que la atención debería ser por un solo psiquiatra; $75.8 \%$ afirma que el trato que reciben es bueno y $75.7 \%$ considera satisfactoria la atención que recibe (cuadro IV). 


\section{Discusión}

Los resultados de este estudio nos permitieron conocer que el alto nivel de EE por el familiar más cercano al paciente, el conocimiento que el paciente y su familiar tienen respecto de la enfermedad mental, y la relación insatisfactoria médico-paciente, son factores psicosociales que estuvieron presentes y que se relacionaron con los frecuentes reingresos a hospitalización de pacientes con psicosis orgánica.

Más de la mitad de los familiares a quienes se les aplicó la entrevista grabada presentaron niveles altos de emoción expresada, con frecuente sobreinvolucramiento emocional y críticas que producen sentimientos negativos hacia el paciente, los cuales pueden considerarse factores de riesgo de recaídas, que en los pacientes de la muestra se presentaron con mayor frecuencia de las que corresponden a la evolución propia de la enfermedad. Además, se observó que son pacientes que conviven diariamente con familiares que presentan niveles altos de emoción expresada; esto corrobora lo que la literatura indica en cuanto a que el registro más alto de recaídas (92\%) se encuentra en pacientes que permanecen más de 35 horas por semana con parientes que presentan alta $\mathrm{EE}^{14}$ debido a que el contacto cara a cara entre el paciente y el familiar propicia un sobreinvolucramiento emocional, perjudica la convivencia y representa un factor de estrés que invariablemente exacerba la sintomatología.

Al comparar la opinión afectiva que el paciente tiene de su familiar con la emoción expresada por el pariente, se encontró una gran discrepancia, pues mientras el paciente considera que el trato que le brinda su familiar y los sentimientos que ese trato le produce son positivos, la entrevista grabada demostró que el paciente es víctima de críticas, relaciones negativas y

Cuadro II

Aspectos de la enfermedad MENTAL CONOCIDOS POR EL famiLiar. México, D.F., Instituto Mexicano del Seguro Social, 1993-1994

Aspectos de la enfermedad mental

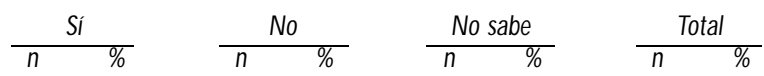

Conoce las características de la enfermedad de su paciente La enfermedad de su paciente es incurable y sólo se controla ${ }^{\ddagger}$, Tomando el medicamento, su paciente se curará totalmente ${ }^{\ddagger}$ A pesar de estar controlada se pueden presentar recaídas Se siente responsable de la enfermedad de su paciente

\begin{tabular}{|c|c|c|c|c|c|c|c|c|}
\hline & \multicolumn{2}{|c|}{ Sí } & \multicolumn{2}{|c|}{ No } & \multicolumn{2}{|c|}{ No sabe } & \multicolumn{2}{|c|}{ Total } \\
\hline & $n$ & $\%$ & $n$ & $\%$ & $\mathrm{n}$ & $\%$ & $\mathrm{n}$ & $\%$ \\
\hline & 18 & $54.5^{*}$ & 11 & 33.4 & 4 & 12.1 & 33 & 100 \\
\hline ola $a^{\ddagger}$, & 24 & 72.7 & 7 & 21.2 & 2 & 6.1 & 33 & 100 \\
\hline$e^{\ddagger}$ & 9 & 27.3 & 22 & 66.7 & 2 & 6.1 & 33 & 100 \\
\hline & 30 & 90.9 & 0 & 0 & 3 & 9.1 & 33 & 100 \\
\hline & 8 & 24.2 & 25 & 75.8 & 0 & 0 & 33 & 100 \\
\hline
\end{tabular}

* Porcentajes de respuestas múltiples

$\ddagger\left(\chi^{2}=18.861, \mathrm{gl} 4, p<0.001\right)$

$\S\left(\chi^{2}=14.652, \mathrm{gl} 4, \mathrm{p}<0.005\right)$

Cuadro III

Concepción del paciente sobre su enfermedad. México, D.F., Instituto Mexicano del Seguro Social, 1993-1994

\begin{tabular}{|c|c|c|c|c|c|c|c|c|}
\hline \multirow[b]{3}{*}{ Concepción de la enfermedad } & \multicolumn{2}{|c|}{ Sí } & \multicolumn{2}{|c|}{ No } & \multicolumn{2}{|c|}{ No sabe } & \multicolumn{2}{|c|}{ Total } \\
\hline & $\mathrm{n}$ & $\%$ & $n$ & $\%$ & $\mathrm{n}$ & $\%$ & $\mathrm{n}$ & $\%$ \\
\hline & & & & & & & & \\
\hline Su enfermedad es incurable f $^{\ddagger}$ & 8 & $24.2^{*}$ & 18 & 54.5 & 7 & 21.3 & 33 & 100 \\
\hline Volverá a estar como antes de enfermarse ${ }^{\ddagger}$ & 18 & 54.5 & 10 & 30.3 & 5 & 15.2 & 33 & 100 \\
\hline Siempre va a estar así & 5 & 15.2 & 22 & 66.6 & 6 & 18.2 & 33 & $\overline{100}$ \\
\hline Conocimientos respecto al medicamento & & & & & & & & \\
\hline Se curará totalmente con el medicamento & 17 & $51.5^{*}$ & 9 & 27.3 & 7 & 21.2 & 33 & 100 \\
\hline Deberá tomar los medicamentos de por vida & 13 & 39.4 & 12 & 36.4 & 8 & 24.2 & 33 & 100 \\
\hline Se hará adicto a los medicamentos & 11 & 33.3 & 16 & 48.5 & 6 & 18.2 & 33 & 100 \\
\hline
\end{tabular}




\section{Cuadro IV \\ Características de la consulta médica. México, D.F., Instituto Mexicano del Seguro SOCIAL, 1993-1994}

\begin{tabular}{lcc}
$\begin{array}{l}\text { Tiempo de asistir a consulta } \\
\text { De } 2 \text { a } 7 \text { años } 11 \text { meses }\end{array}$ & $n$ & $\%$ \\
\hline De 8 a 13 años 11 meses & 15 & 45.4 \\
\hline De 14 a 19 años 11 meses & 10 & 30.3 \\
\hline De 20 a 25 años 11 meses & 5 & 15.2 \\
\hline $\begin{array}{l}\text { Frecuencia de las consultas } \\
\text { C ada } 15 \text { días }\end{array}$ & 3 & 9.1 \\
\hline Cada mes & 1 & \\
\hline De mes y medio a dos & 28 & 84.0 \\
\hline De dos meses y medio a tres & 2 & 6.1 \\
\hline $\begin{array}{l}\text { Tiempo de cada consulta } \\
\text { De } 5 \text { a } 10 \text { minutos }\end{array}$ & 2 & 6.1 \\
\hline De 15 a 20 minutos & & \\
\hline De media hora a tres cuartos de hora & 9 & 27.3 \\
\hline De una hora & 9 & 27.3 \\
\hline $\begin{array}{l}\text { Número de psiquiatras que lo han atendido } \\
\text { De } 1 \text { a } 3\end{array}$ & 6 & 27.3 \\
\hline De 4 a 6 & 24 & 18 \\
\hline De 7 a 10 & 4 & 12.7 \\
\hline & 5 & 15.2
\end{tabular}

\begin{tabular}{lcc}
$\begin{array}{l}\text { Trato que le brinda el psiquiatra } \\
\text { Bueno }\end{array}$ & 25 & 75.8 \\
\hline Regular & 7 & 21.2 \\
\hline Malo & 1 & 3.0
\end{tabular}

0 pinión del paciente respecto de la atención psiquiátrica

\begin{tabular}{|c|c|c|c|c|c|c|c|c|}
\hline & & Sí & $\mathrm{N}$ & 0 & No & sabe & & tal \\
\hline & $\mathrm{n}$ & $\%$ & $\bar{n}$ & $\%$ & $\bar{n}$ & $\%$ & $\bar{n}$ & $\%$ \\
\hline Atención psiquiátrica & & & & & & & & \\
\hline El tiempo de consulta & & & & & & & & \\
\hline es suficiente $e^{\ddagger}$ & 22 & $66.7^{*}$ & 10 & 30.3 & 1 & 3.0 & 33 & 100 \\
\hline Está de acuerdo con & & & & & & & & \\
\hline los cambios de psiquiatra & 13 & 39.4 & 15 & 45.5 & 5 & 15.1 & 33 & 100 \\
\hline La atención debe ser por & & & & & & & & \\
\hline un solo psiquiatra & 23 & 69.7 & 8 & 24.3 & 2 & 6.0 & 33 & 100 \\
\hline Considera satisfactoria & & & & & & & & \\
\hline la atención & 25 & 75.7 & 8 & 24.3 & 0 & 0 & 33 & 100 \\
\hline
\end{tabular}

excesivo involucramiento emocional por parte del familiar.

El instrumento más reciente para evaluar EE en el familiar más cercano al paciente es "La Muestra de Conversación de Cinco Minutos", entrevista que ha sido aplicada en diferentes países a pacientes con diversos padecimientos como esquizofrenia, enfermedad bipolar, depresión, lesión cerebral, etcétera.

Por parte del paciente y de su familiar existe un gran desconocimiento del nombre y características de la enfermedad que se padece; la mayoría de los sujetos de la muestra no acepta tener una enfermedad mental, piensan que los problemas emocionales han sido la causa de la situación en que se encuentran, consideran que su enfermedad es curable, que no siempre van a estar así y que tomando los medicamentos volverán a estar como antes de enfermarse, por lo tanto no deberán tomarlos de por vida; sin embargo, se advierte que los familiares tienen más conciencia de enfermedad, ya que ellos advierten que la enfermedad de su paciente es incurable y con los medicamentos sólo podrá ser controlada y que, a pesar de ello, podrá presentar recaídas.

En cuanto a la percepción que el paciente tiene de su ambiente familiar podemos mencionar que quienes consideraron a sus padres positivamente tendieron a experimentar un curso más leve de la enfermedad, a diferencia de aquéllos que percibieron a sus padres negativamente; en éstos se observó un curso más severo del padecimiento. ${ }^{15}$

Otros investigadores encontraron que la percepción que los pacientes tienen de sus familiares puede ayudar a mejorar o empeorar el estado del paciente, es decir, que la probabilidad de tener una recaída aumenta o disminuye si el familiar es percibido negativa o positivamente. ${ }^{16}$ Esta percepción está altamente relacionada con las actitudes que los familiares describen de su paciente. Otros hallazgos han mostrado que pacientes que sufren recaídas provienen de padres inseguros, vulnerables o que no tenían conocimiento de cómo eran percibidos por su familiar enfermo, siendo la percepción de éstos desfavorable. ${ }^{17}$

El desconocimiento de la enfermedad por el paciente origina que éste no le dé importancia a los medicamentos y abandone el tratamiento cuando se siente bien, o cuando, por los efectos colaterales, cree que "la medicina le está haciendo daño". Probablemente este desconocimiento se debe al poco énfasis que se le da a los beneficios del medicamento, así como la poca información sobre los efectos secundarios y las características de la enfermedad en la consulta psiquiátrica.

La relación médico-paciente se establece en forma satisfactoria, la percepción que tiene el paciente de su psiquiatra es buena, pero al parecer la comunicación no es lo suficientemente explícita para que se forme en el paciente conciencia de enfermedad y apego al tratamiento terapéutico. 


\section{Agradecimientos}

Con el más profundo respeto y admiración se agradece a la doctora María Elena Medina Mora el tiempo tan valioso que concedió en la orientación y revisión de este trabajo.

Al doctor Alberto Martínez Ruiz, Director del Hospital Psiquiátrico con UMF No. 10 por haber permitido realizar en esa institución la presente investigación.

A los pacientes y familiares que con la mayor disposición aceptaron colaborar y sin los cuales no hubiera sido posible realizar este trabajo.

\section{Referencias}

1. Medina-Mora ME, Rascón-Gasca ML,Tapia CR, Mariño ME, Juárez F, Caraveo AJ.Trastornos emocionales en población urbana. Resultados de un estudio nacional. AN ALES. Instituto Mexicano de Psiquiatría, 1992.

2. Magaña AB, Goldstein MJ, Karmo M. A Brief method for assessing expresses emotion in relatives of psychiatric patients. Psychiatriy res 1986; 17:203-212.

3. Brown GW , C arstairs GM, Topping GC. The post hospital adjustment of chronic mental patients. Lancet 1958;2:685-689.

4. Gottschalk LA, Gleser GC. The Measurement of psychological states through the content analysis of verbal behaviour. Berkeley (CA):University of California Press, 1969.

5. Masanet MJ, Lacruz M, A sencio A, Belver F, Montero I. Clima emocional familiar y perfil clínico en la esquizofrenia. Actas Luso-Españolas de $\mathrm{N}$ eurología, Psiquiatría y Ciencias A fines 1997;25(3):159-166.
6. W einer $B$, Perry P, Magnusson J.An attributional analysis of reactions to stigmas. J Pers Soc Psychol 1988;55(5):738-748.

7. Murillo N . The Mexican-American family. En: Hernández CA, Haug MJ, W agner N N . eds. Chicano: Social and psychological perspectives: St. Louis (MO): CV Mosby 1976:13-24.

8. Jenkins JH, Karno M, D e La Selva A, Santana F. Expressed emotion in cross-cultural context: Familial responses to schizophrenic illness among Mexican-A mericans. En: Goldstein I, H, Hahlweg K, ed.Treatment of schizophrenia: Family assesment and intervention. N uevaYork (N Y): SpringerVerlag:35-49.

9.W eisman A, López S. Family values, religiosidad, and emotional reactions to schizo phrenia in Mexican and Anglo-American cultures. Family Process, 1996:35:227-237.

10. Lain-Entralgo P. La relación médico-enfermo. Madrid:Alianza Editorial, 1983.

11. Puente-Silva F.Adherencia terapéutica. Implicaciones para México. Simposium A dherenciaTerapéutica XIII Congreso Internacional de Psicología; 1984 sept 7;Acapulco (G ro), México.

12. Ling J, Basafeld P.Tender puentes entre médicos y enfermos. Foro Mundial de la Salud 1989;10: 29-30.

13. Rascón GML, Díaz MLR, López JL, Reyes RC. N ecesidad de dar atención a los familiares responsables del cuidado de pacientes esquizofrénicos. Salud Mental 1997; (Supl 2):

14. Holey-JILL M. Expressed emotion: A review of the critical literature. Clin Psycol Rev 1986;5:119-139.

15.W arner R,Atkinson M.The relationship betwen schizophrenic patiens, perceptions of their parents and the course of their illness. Bri J Psychiatry 1988;153:344-353.

16. Lebell M, Marder RE, Mintz J, Mintz IL, Tompson M, W shing W et al. Patients, perceptions family emotional climate and outcome in schizophrenia. Br J Psychiatry 1993;162: 751-754.

17. Scott R, Fagin L,W inter D.The importance of the role of the patients in the outcome of schizophrenia. Br J Psychiatry 1993;163:62-68, 